\title{
On Fuzzy Improper Integral and Its Application for Fuzzy Partial Differential Equations
}

\author{
ElHassan ElJaoui and Said Melliani \\ Department of Mathematics, University of Sultan Moulay Slimane, P.O. Box 523, 23000 Beni Mellal, Morocco \\ Correspondence should be addressed to ElHassan ElJaoui; eljaouihass@gmail.com
}

Received 31 October 2015; Revised 20 December 2015; Accepted 3 January 2016

Academic Editor: Najeeb A. Khan

Copyright ( 2016 E. ElJaoui and S. Melliani. This is an open access article distributed under the Creative Commons Attribution License, which permits unrestricted use, distribution, and reproduction in any medium, provided the original work is properly cited.

We establish some important results about improper fuzzy Riemann integrals; we prove some properties of fuzzy Laplace transforms, which we apply for solving some fuzzy linear partial differential equations of first order, under generalized Hukuhara differentiability.

\section{Introduction}

Wu introduced in [1] the improper fuzzy Riemann integral and presented some of its elementary properties; then he studied numerically this kind of integrals.

This notion was exploited by certain researchers to study fuzzy differential equations (FDEs) of first or second order utilizing fuzzy Laplace transform, namely, by Allahviranloo and Ahmadi in [2], then by Salahshour et al. (see [3,4]), and by ElJaoui et al. in [5].

The objective of this paper is to study the improper fuzzy Riemann integrals by establishing some important results about the continuity and the differentiability of a fuzzy improper integral depending on a given parameter.

These results are then employed to prove some fuzzy Laplace transform's properties, which we use to solve fuzzy partial differential equations (FPDEs).

The organization of the remainder of this work is as follows. Section 2 is reserved for preliminaries. In Section 3, the main results are proved and new properties of fuzzy Laplace transform are investigated. Then, in Section 4, the procedure for solving first-order FPDEs by fuzzy Laplace transform is proposed. Section 5 deals with some numerical examples. In Section 6, we present conclusion and a further research topic.

\section{Preliminaries}

By $P_{c}(\mathbb{R})$ we meant the set of all nonempty compact convex subsets of $\mathbb{R}$, which is endowed with the usual addition and scalar multiplication. Denote (see [6])

$$
E=\{\mu: \mathbb{R} \longrightarrow[0,1] \mid \mu \text { verifies (1) - (4) below }\},
$$

where

(1) $\mu$ is normal; that is, $\exists t \in \mathbb{R}$ for which $\mu(t)=1$,

(2) $\mu$ is convex in the fuzzy sense,

(3) $\mu$ is upper semicontinuous,

(4) the closure of its support supp $\mu=\{t \in \mathbb{R} \mid \mu(t)>0\}$ is compact.

For $0<\alpha \leq 1,[\mu]^{\alpha}=\{t \in \mathbb{R} \mid \mu(t) \geq \alpha\}$ denotes the $\alpha$-level set of $\mu \in E$.

Then, it is obvious that $[\mu]^{\alpha} \in P_{c}(\mathbb{R})$ for all $\mu \in E, 0 \leq \alpha \leq$ 1 , and

$$
\begin{aligned}
{\left[\mu_{1}+\mu_{2}\right]^{\alpha} } & =\left[\mu_{1}\right]^{\alpha}+\left[\mu_{2}\right]^{\alpha}, \\
{[k \mu]^{\alpha} } & =k[\mu]^{\alpha} .
\end{aligned}
$$

Let $D: E \times E \rightarrow[0, \infty)$ be a function which is defined by the identity

$$
D\left(\mu_{1}, \mu_{2}\right)=\sup _{0 \leq \alpha \leq 1} d\left(\left[\mu_{1}\right]^{\alpha},\left[\mu_{2}\right]^{\alpha}\right),
$$


where $d$ is the Hausdorff distance defined in $P_{c}(\mathbb{R})$. Then, it is clear that $(E, D)$ is a complete metric space (for more details about the metric $D$ see [7]).

Definition 1 (see [2]). One defines a fuzzy number $v$ in parametric form as a couple $(\underline{v}, \bar{v})$ of mappings $\underline{v}(\alpha)$ and $\bar{v}(\alpha)$, $0 \leq \alpha \leq 1$, verifying the following properties:

(1) $\underline{v}(\alpha)$ is bounded increasing left continuous in ]0,1] and right continuous at 0 .

(2) $\bar{v}(\alpha)$ is bounded decreasing left continuous in $] 0,1]$ and right continuous at 0 .

(3) $\underline{v}(\alpha) \leq \bar{v}(\alpha)$ for all $0 \leq \alpha \leq 1$.

The following identity holds true (see [8]):

$$
D\left(\mu_{1}, \mu_{2}\right)=\sup _{0 \leq \alpha \leq 1} \max \left\{\left|\underline{\mu}_{1}^{\alpha}-\underline{\mu}_{2}^{\alpha}\right|,\left|\bar{\mu}_{1}^{\alpha}-\bar{\mu}_{2}^{\alpha}\right|\right\} .
$$

Theorem 2 (see [1]). One considers a fuzzy valued function $F(x)=(\underline{F}(x, \alpha), \bar{F}(x, \alpha))$ defined on $[a, \infty[$. Suppose that, for all fixed $\alpha \in[0,1]$, the crisp functions $\underline{F}(x, \alpha), \bar{F}(x, \alpha)$ are integrable on $[a, b]$, for every $b \geq a$, and that there exist two positive constants $\underline{K}(\alpha)$ and $\bar{K}(\alpha)$ such that $\int_{a}^{b}|\underline{F}(x, \alpha)| d x \leq$ $\underline{K}(\alpha)$ and $\int_{a}^{b}|\bar{F}(x, \alpha)| d x \leq \bar{K}(\alpha)$ for every $b \geq a$. Then $F(x)$ is fuzzy Riemann integrable (in the sense of $W u$ ) on $[a, \infty[$, its improper fuzzy integral $\int_{a}^{\infty} F(x) d x \in E$, and

$$
\int_{a}^{\infty} F(x) d x=\left(\int_{a}^{\infty} \underline{F}(x, \alpha) d x, \int_{a}^{\infty} \bar{F}(x, \alpha) d x\right) .
$$

For $\mu_{1}, \mu_{2} \in E$, if there exists an element $\mu_{3} \in E$ such that $\mu_{3}=\mu_{1}+\mu_{2}$, then $\mu_{3}$ is called the Hukuhara difference of $\mu_{1}$ and $\mu_{2}$, which we denote by $\mu_{1} \ominus \mu_{2}$.

Definition 3 (see [2]). A mapping $F:(a, b) \rightarrow E$ is said to be strongly generalized differentiable at $x \in(a, b)$, if there exists $F^{\prime}(x) \in E$, such that

(i) for all $h>0$ being very small, there exist $F(x+h) \ominus$ $F(x) ; F(x) \ominus F(x-h)$; and the limits

$$
\begin{aligned}
& \lim _{h \rightarrow 0^{+}} \frac{F(x+h) \ominus F(x)}{h}=\lim _{h \rightarrow 0^{+}} \frac{F(x) \ominus F(x-h)}{h} \\
& =F^{\prime}(x),
\end{aligned}
$$

or

(ii) for all $h>0$ being very small, there exist $F(x) \ominus F(x+$ $h) ; F(x-h) \ominus F(x)$; and the limits

$$
\begin{aligned}
& \lim _{h \rightarrow 0^{+}} \frac{F(x) \ominus F(x+h)}{(-h)}=\lim _{h \rightarrow 0^{+}} \frac{F(x-h) \ominus F(x)}{(-h)} \\
& \quad=F^{\prime}(x)
\end{aligned}
$$

or

(iii) for all $h>0$ being very small, there exist $F(x+h) \ominus$ $F(x) ; F(x-h) \ominus F(x)$; and the limits

$$
\begin{aligned}
& \lim _{h \rightarrow 0^{+}} \frac{F(x+h) \ominus F(x)}{h}=\lim _{h \rightarrow 0^{+}} \frac{F(x-h) \ominus F(x)}{(-h)} \\
& =F^{\prime}(x),
\end{aligned}
$$

or

(iv) for all $h>0$ being very small, there exist $F(x) \ominus F(x+$ $h) ; F(x) \ominus F(x-h)$; and the limits

$$
\begin{aligned}
& \lim _{h \rightarrow 0^{+}} \frac{F(x) \ominus F(x+h)}{(-h)}=\lim _{h \rightarrow 0^{+}} \frac{F(x) \ominus F(x-h)}{h} \\
& =F^{\prime}(x) .
\end{aligned}
$$

The next theorem permits us to consider only case (i) or case (ii) of Definition 3 almost everywhere in the domain of the mappings studied.

Theorem 4 (see [9]). If $F:(a, b) \rightarrow E$ is a strongly generalized differentiable function on $(a, b)$ in the sense of Definition 3, (iii) or (iv), then $F^{\prime}(x) \in \mathbb{R}$ for each $x \in(a, b)$.

Theorem 5 (see, e.g., [10]). We consider a fuzzy function $F$ : $\mathbb{R} \rightarrow E$ which is represented by $F(x)=(\underline{F}(x, \alpha), \bar{F}(x, \alpha))$, for all $\alpha \in[0,1]$ :

(1) If $F$ is (i)-differentiable, then the crisp functions $\underline{F}(x, \alpha)$ and $\bar{F}(x, \alpha)$ are differentiable and $F^{\prime}(x)=$ $\left(F^{\prime}(x, \alpha), \bar{F}^{\prime}(x, \alpha)\right)$.

(2) If $F$ is (ii)-differentiable, then the crisp functions $\underline{F}(x, \alpha)$ and $\bar{F}(x, \alpha)$ are differentiable and $F^{\prime}(x)=$ $\left(\bar{F}^{\prime}(x, \alpha), \underline{F}^{\prime}(x, \alpha)\right)$.

Definition 6 (see [2]). If $F:[0, \infty[\rightarrow E$ is a continuous mapping such that $e^{-s x} F(x)$ is fuzzy Riemann integrable on $\left[0, \infty\left[\right.\right.$ then $\int_{0}^{\infty} e^{-s x} F(x) d x$ is called the fuzzy Laplace transform of $F$ which one denotes by

$$
\mathbf{L}[F(x)]=\int_{0}^{\infty} e^{-s x} F(x) d x, \quad s>0 .
$$

Denote by $\mathscr{L}(k(x))$ the classical Laplace transform of a crisp function $k(x)$, and then

$$
\mathbf{L}[F(x)]=(\mathscr{L}(\underline{F}(x, \alpha)), \mathscr{L}(\bar{F}(x, \alpha))) .
$$

Theorem 7 (see [2]). Let $F:[0, \infty[\rightarrow E$ be a fuzzy valued function and $F^{\prime}$ its derivative on $[0, \infty[$. Then, if $F$ is (i)differentiable

$$
\mathbf{L}\left[F^{\prime}(x)\right]=s \mathbf{L}[F(x)] \ominus F(0)
$$

or if $F$ is (ii)-differentiable

$$
\mathbf{L}\left[F^{\prime}(x)\right]=(-F(0)) \ominus(-s) \mathbf{L}[F(x)]
$$

provided that the Laplace transforms of $F$ and $F^{\prime}$ exist. 


\section{Continuity and Differentiability of Fuzzy Improper Integral}

In this section, $I$ denotes one of the intervals, $]-\infty, b]$ or $[b, \infty[$ or $]-\infty, \infty[$, where $b \in \mathbb{R}, J$ denotes another interval, and $A$ is a nonempty subset of $\mathbb{R}$.

Lemma 8. Let $f_{1}(t), f_{2}(t)$ be two fuzzy valued functions, which are fuzzy Riemann integrable on $I$, in the sense of $W u$ (see [1]), such that the real function $D\left(f_{1}(t), f_{2}(t)\right)$ is integrable on $I$, and then

$$
D\left(\int_{I} f_{1}(t) d t, \int_{I} f_{2}(t) d t\right) \leq \int_{I} D\left(f_{1}(t), f_{2}(t)\right) d t .
$$

$$
\begin{aligned}
D\left(\int_{I} f_{1}(t) d t, \int_{I} f_{2}(t) d t\right) & =\sup _{0 \leq \alpha \leq 1} \max \left\{\left|\int_{I} \underline{f}_{1}(t, \alpha)-\underline{f}_{2}(t, \alpha) d t\right|,\left|\int_{I} \bar{f}_{1}(t, \alpha) d t-\bar{f}_{2}(t, \alpha) d t\right|\right\} \\
& \leq \sup _{0 \leq \alpha \leq 1} \max \left\{\int_{I}\left|\underline{f}_{1}(t, \alpha)-\underline{f}_{2}(t, \alpha)\right| d t, \int_{I}\left|\bar{f}_{1}(t, \alpha)-\bar{f}_{2}(t, \alpha)\right| d t\right\} \\
& \leq \sup _{0 \leq \alpha \leq 1} \int_{I} \max \left\{\left|\underline{f}_{1}(t, \alpha)-\underline{f}_{2}(t, \alpha)\right|,\left|\bar{f}_{1}(t, \alpha)-\bar{f}_{2}(t, \alpha)\right|\right\} d t \\
& \leq \int_{I} \sup _{0 \leq \alpha \leq 1} \max \left\{\left|\underline{f}_{1}(t, \alpha)-\underline{f}_{2}(t, \alpha)\right|,\left|\bar{f}_{1}(t, \alpha)-\bar{f}_{2}(t, \alpha)\right|\right\} d t=\int_{I} D\left(f_{1}(t), f_{2}(t)\right) d t .
\end{aligned}
$$

Theorem 9. Let $F(x, t): A \times I \rightarrow E$ be a fuzzy function, satisfying the following conditions:

$\left(H_{1}\right)$ For all $x \in A, t \mapsto F(x, t)$ is continuous on $I$.

$\left(H_{2}\right)$ For each $t \in I, x \mapsto F(x, t)$ is continuous on $A \subset \mathbb{R}$.

$\left(H_{3}\right)$ For all $\alpha \in[0,1]$ there exist a couple of nonnegative, continuous crisp functions $\varphi_{\alpha}(t)$ and $\psi_{\alpha}(t)$, which are integrable on I verifying, for all $x \in A, t \in I$ :

$$
\begin{aligned}
& |\underline{F}(x, t, \alpha)| \leq \varphi_{\alpha}(t), \\
& |\bar{F}(x, t, \alpha)| \leq \psi_{\alpha}(t) .
\end{aligned}
$$

Therefore, the fuzzy mapping $\phi(x)=\int_{I} F(x, t) d t$ is continuous on $A$.

Proof. Let $x \in A$ and let $\left\{x_{k}\right\}_{k=1}^{\infty}$ be a sequence of elements of $A$, which converges to $x$ as $k \rightarrow \infty$. For $k \in \mathbb{N}, t \in I$, and $\alpha \in[0,1]$, we have

$$
\begin{aligned}
& \underline{F}\left(x_{k}, t, 0\right) \leq \underline{F}\left(x_{k}, t, \alpha\right) \leq \underline{F}\left(x_{k}, t, 1\right), \\
& \bar{F}\left(x_{k}, t, 1\right) \leq \bar{F}\left(x_{k}, t, \alpha\right) \leq \bar{F}\left(x_{k}, t, 0\right) .
\end{aligned}
$$

Thus

$$
\begin{aligned}
\left|\underline{F}\left(x_{k}, t, \alpha\right)\right| & \leq \max \left\{\left|\underline{F}\left(x_{k}, t, 1\right)\right|,\left|\underline{F}\left(x_{k}, t, 0\right)\right|\right\} \\
& \leq \max \left\{\varphi_{0}(t), \varphi_{1}(t)\right\}=g(t), \\
\left|\bar{F}\left(x_{k}, t, \alpha\right)\right| & \leq \max \left\{\left|\bar{F}\left(x_{k}, t, 1\right)\right|,\left|\bar{F}\left(x_{k}, t, 0\right)\right|\right\} \\
& \leq \max \left\{\psi_{0}(t), \psi_{1}(t)\right\}=h(t) .
\end{aligned}
$$

By tending $k \rightarrow \infty$ and using assumption $\left(\mathrm{H}_{2}\right)$, we obtain

$$
\begin{aligned}
& |\underline{F}(x, t, \alpha)| \leq \max \left\{\varphi_{0}(t), \varphi_{1}(t)\right\}=g(t), \\
& |\bar{F}(x, t, \alpha)| \leq \max \left\{\psi_{0}(t), \psi_{1}(t)\right\}=h(t) .
\end{aligned}
$$

Therefore

$$
\begin{aligned}
D & \left(F\left(x_{k}, t\right), F(x, t)\right) \\
& =\sup _{0 \leq \alpha \leq 1} \max \left\{\left|\underline{F}\left(x_{k}, t, \alpha\right)-\underline{F}(x, t, \alpha)\right|,\right. \\
& \left.\left|\bar{F}\left(x_{k}, t, \alpha\right)-\bar{F}(x, t, \alpha)\right|\right\}, \\
D & \left(F\left(x_{k}, t\right), F(x, t)\right) \leq 2(g(t)+h(t)) .
\end{aligned}
$$

From $\left(H_{1}\right)$ and $\left(H_{3}\right)$, we deduce that the mappings $g(t), h(t)$, and $D\left(F\left(x_{k}, t\right), F(x, t)\right)$ are all integrable on $I$.

On the other hand, we get the following inequality from Lemma 8:

$$
\begin{array}{r}
D\left(\int_{I} F\left(x_{k}, t\right) d x, \int_{I} F(x, t) d x\right) \\
\quad \leq \int_{I} D\left(F\left(x_{k}, t\right), F(x, t)\right) d x .
\end{array}
$$

That is,

$$
D\left(\phi\left(x_{k}\right), \phi(x)\right) \leq \int_{I} D\left(F\left(x_{k}, t\right), F(x, t)\right) d x .
$$

By assumption $\left(H_{2}\right)$, we have $D\left(F\left(x_{k}, t\right), F(x, t)\right) \rightarrow 0$ as $k \rightarrow$ $\infty$. 
So, by the dominated convergence theorem, $\int_{I} D\left(F\left(x_{k}, t\right), F(x, t)\right) d x \rightarrow 0$ as $k \rightarrow \infty$.

From inequality (22), we deduce that $\phi\left(x_{k}\right) \rightarrow \phi(x)$ as $k \rightarrow \infty$.

Consequently, $\phi$ is continuous on $A$.

Lemma 10. One considers two fuzzy valued functions $f_{1}(t)$, $f_{2}(t): I \rightarrow E$, which are fuzzy Riemann integrable on $I$ (in the sense of $W u)$, such that $f_{1}(t) \ominus f_{2}(t)$ exists for all $t \in I$, then $f_{1}(t) \ominus f_{2}(t)$ is fuzzy Riemann integrable on $I$, the Hukuhara difference $\int_{I} f_{1}(t) d t \ominus \int_{I} f_{2}(t) d t$ is well defined, and

$$
\int_{I}\left(f_{1}(t) \ominus f_{2}(t)\right) d x=\int_{I} f_{1}(t) d t \ominus \int_{I} f_{2}(t) d t .
$$

Proof. Let $k(t)=f_{1}(t) \ominus f_{2}(t)$; that is, $f_{1}(t)=f_{2}(t)+k(t)$. It is clear that there exist positive constants $\underline{K}\left(\alpha, f_{1}\right), \bar{K}\left(\alpha, f_{1}\right)$, $\underline{K}\left(\alpha, f_{2}\right)$, and $\bar{K}\left(\alpha, f_{2}\right)$ such that, for all $a \leq b$ in $I$, we have

$$
\begin{aligned}
& \int_{a}^{b}\left|\underline{f}_{1}(t, \alpha)\right| d t \leq \underline{K}\left(\alpha, f_{1}\right), \\
& \int_{a}^{b}\left|\bar{f}_{1}(t, \alpha)\right| d t \leq \bar{K}\left(\alpha, f_{1}\right), \\
& \int_{a}^{b}\left|\underline{f}_{2}(t, \alpha)\right| d t \leq \underline{K}\left(\alpha, f_{2}\right), \\
& \int_{a}^{b}\left|\bar{f}_{2}(t, \alpha)\right| d t \leq \bar{K}\left(\alpha, f_{2}\right) .
\end{aligned}
$$

Hence

$$
\begin{aligned}
\int_{a}^{b}|\underline{k}(t, \alpha)| d t & =\int_{a}^{b}\left|\underline{f}_{1}(t, \alpha)-\underline{f}_{2}(t, \alpha)\right| d t \\
& \leq \underline{K}\left(\alpha, f_{1}\right)+\underline{K}\left(\alpha, f_{2}\right)
\end{aligned}
$$

and similarly

$$
\begin{aligned}
\int_{a}^{b}|\bar{k}(t, \alpha)| d t & =\int_{a}^{b}\left|\bar{f}_{1}(t, \alpha)-\bar{f}_{2}(t, \alpha)\right| d t \\
& \leq \bar{K}\left(\alpha, f_{1}\right)+\bar{K}\left(\alpha, f_{2}\right) .
\end{aligned}
$$

Then from Theorem 2, $k(t)$ is fuzzy Riemann integrable on $I$.

By "linearity" of the fuzzy integral, we get

$$
\int_{I} f_{1}(t) d t=\int_{I} f_{2}(t) d t+\int_{I} k(t) d t
$$

Thus, $\int_{I} f_{1}(t) d t \ominus \int_{I} f_{2}(t) d t$ exists and $\int_{I} f_{1}(t) d t \ominus \int_{I} f_{2}(t) d t=$ $\int_{I} k(t) d t$.

Theorem 11. One considers a fuzzy valued function $F(x, t)$ : $J \times I \rightarrow E$, verifying the following assumptions:

$\left(A_{1}\right)$ For all $x \in J, t \mapsto F(x, t)$ is continuous and fuzzy Riemann integrable on $I$.

$\left(A_{2}\right)$ For all $t \in I, x \mapsto F(x, t)$ is (i)-differentiable on the interval $J$.
$\left(A_{3}\right)$ For all $x \in J, t \mapsto(\partial F / \partial x)(x, t)$ is continuous on $I$.

$\left(A_{4}\right)$ For all $t \in I, x \mapsto(\partial F / \partial x)(x, t)$ is continuous on $J$.

$\left(A_{5}\right)$ For all $\alpha \in[0,1]$ there exist a couple of continuous crisp functions $\varphi_{\alpha}(t)$ and $\psi_{\alpha}(t)$, which are integrable on $I$ verifying, for all $x \in J, t \in I$ :

$$
\begin{aligned}
& \left|\frac{\partial \underline{F}}{\partial x}(x, t, \alpha)\right| \leq \varphi_{\alpha}(t), \\
& \left|\frac{\partial \bar{F}}{\partial x}(x, t, \alpha)\right| \leq \psi_{\alpha}(t) .
\end{aligned}
$$

Therefore, the fuzzy mapping $\phi(x)=\int_{I} F(x, t) d t$ is (i)-differentiable on $J$ and

$$
\phi^{\prime}(x)=\int_{I} \frac{\partial F}{\partial x}(x, t) d t, \quad \forall x \in J .
$$

Moreover, if one replaces assumption $\left(A_{2}\right)$ by the alternative condition

$$
\left(A_{2}^{\prime}\right) \text { for all } t \in I, x \mapsto F(x, t) \text { is (ii)-differentiable on } J \text {, }
$$

then the fuzzy function $\phi(x)$ is (ii)-differentiable on J and (29) remains true.

Proof. Assume that $\left(A_{1}\right)-\left(A_{5}\right)$ hold true. Let $x \in J, \xi_{0}>0$ being very small, and define the auxiliary functions

$$
\begin{aligned}
& g_{1}(\xi, t)= \begin{cases}\frac{F(x+\xi, t) \ominus F(x, t)}{\xi}, & \left.\xi \in] 0, \xi_{0}\right] \\
\frac{\partial F}{\partial x}(x, t), & \xi=0,\end{cases} \\
& g_{2}(\xi, t)= \begin{cases}\frac{F(x, t) \ominus F(x-\xi, t)}{\xi}, & \left.\xi \in] 0, \xi_{0}\right] \\
\frac{\partial F}{\partial x}(x, t), & \xi=0 .\end{cases}
\end{aligned}
$$

For fixed $\left.\xi \in] 0, \xi_{0}\right]$, we have

$$
\begin{aligned}
& \frac{\phi(x+\xi) \ominus \phi(x)}{\xi} \\
& =\frac{1}{\xi}\left(\int_{I} F(x+\xi, t) d t \ominus \int_{I} F(x, t) d t\right) \\
& =\int_{I} \frac{F(x+\xi, t) \ominus F(x, t)}{\xi} d t=\int_{I} g_{1}(\xi, t) d t,
\end{aligned}
$$

where the existence of the Hukuhara differences is ensured by the (i)-differentiability of $x \mapsto F(x, t)$ and by Lemma 10 .

Analogously, we get

$$
\frac{\phi(x) \ominus \phi(x-\xi)}{\xi}=\int_{I} g_{2}(\xi, t) d t .
$$

From assumptions $\left(A_{1}\right)-\left(A_{4}\right)$, we deduce that $g_{1}$ and $g_{2}$ satisfy conditions $\left(H_{1}\right)-\left(H_{2}\right)$ of Theorem 9 . 
On the other hand, using the finite increments theorem, we obtain

$$
\begin{aligned}
\left|\underline{g}_{1}(\xi, t, \alpha)\right| & =\left|\frac{\underline{F}(x+\xi, t, \alpha)-\underline{F}(x, t, \alpha)}{\xi}\right| \\
& \leq \sup _{0 \leq v \leq \xi_{0}}\left|\frac{\partial \underline{F}}{\partial x}(x+v, t, \alpha)\right| \leq \varphi_{\alpha}(t), \\
\left|\bar{g}_{1}(\xi, t, \alpha)\right| & =\left|\frac{\bar{F}(x+\xi, t, \alpha)-\bar{F}(x, t, \alpha)}{\xi}\right| \\
& \leq \sup _{0 \leq v \leq \xi_{0}}\left|\frac{\partial \bar{F}}{\partial x}(x+v, t, \alpha)\right| \leq \psi_{\alpha}(t) .
\end{aligned}
$$

Similarly, we have

$$
\begin{aligned}
\left|\underline{g}_{2}(\xi, t, \alpha)\right| & =\left|\frac{\underline{F}(x, t, \alpha)-\underline{F}(x-\xi, t, \alpha)}{h}\right| \\
& \leq \sup _{0 \leq v \leq \xi_{0}}\left|\frac{\partial \underline{F}}{\partial x}(x-v, t, \alpha)\right| \leq \varphi_{\alpha}(t), \\
\left|\bar{g}_{2}(\xi, t, \alpha)\right| & =\left|\frac{\bar{F}(x, t, \alpha)-\bar{F}(x-\xi, t, \alpha)}{h}\right| \\
& \leq \sup _{0 \leq v \leq \xi_{0}}\left|\frac{\partial \bar{F}}{\partial x}(x-v, t, \alpha)\right| \leq \psi_{\alpha}(t) .
\end{aligned}
$$

Inequalities (33) and (34), which are obviously also true for $\xi=0$, ensure that $g_{1}$ and $g_{2}$ satisfy condition $\left(H_{3}\right)$ of Theorem 9.

Applying the latter theorem, we get

$$
\begin{gathered}
\lim _{\xi \rightarrow 0^{+}} \frac{\phi(x+\xi) \ominus \phi(x)}{\xi}=\int_{I} g_{1}(0, t) d t \\
=\int_{I} \frac{\partial F}{\partial x}(x, t) d t, \\
\lim _{\xi \rightarrow 0^{+}} \frac{\phi(x) \ominus \phi(x-\xi)}{\xi}=\int_{I} g_{2}(0, t) d t \\
=\int_{I} \frac{\partial F}{\partial x}(x, t) d t .
\end{gathered}
$$

Therefore, $\phi$ is (i)-differentiable at $x$ and

$$
\phi^{\prime}(x)=\int_{I} \frac{\partial F}{\partial x}(x, t) d t
$$

The proof under assumption $\left(A_{2}^{\prime}\right)$ instead of $\left(A_{2}\right)$ is similar to the first case.

Theorem 12. One considers a fuzzy function $u(\xi, \tau)$ : $[0, \infty[\times[0, \infty[\rightarrow \quad E$. Suppose that the mapping $F(\xi, \tau)=$ $e^{-s \tau} u(\xi, \tau)$ satisfies assumptions $\left(A_{1}\right)-\left(A_{5}\right)$ above, for all $s \geq s_{0}$ for some $s_{0}>0$.
Let $\mathbf{L}_{\tau}[u(\xi, \tau)]$ or $\mathbf{L}[u(\xi, \tau)]$ (for short) denote the fuzzy Laplace transform of $u(\xi, \tau)$ with respect to the time variable $\tau$. Then

$$
\mathbf{L}_{\tau}\left[u_{\xi}(\xi, \tau)\right]=\frac{\partial}{\partial \xi}\left(\mathbf{L}_{\tau}[u(\xi, \tau)]\right)
$$

Proof. For fixed $s \geq s_{0}$, then using Theorem 11 we have

$$
\begin{aligned}
\mathbf{L}_{\tau}\left[u_{\xi}(\xi, \tau)\right] & =\int_{0}^{\infty} e^{-s \tau} u_{\xi}(\xi, \tau) d \tau=\int_{0}^{\infty} F_{\xi}(\xi, \tau) d \tau \\
& =\frac{\partial}{\partial \xi}\left(\int_{0}^{\infty} F(\xi, \tau) d \tau\right), \\
\mathbf{L}_{\tau}\left[u_{\xi}(\xi, \tau)\right] & =\frac{\partial}{\partial \xi}\left(\mathbf{L}_{\tau}[u(\xi, \tau)]\right) .
\end{aligned}
$$

Theorem 13. Let $u(\xi, \tau)$ be a fuzzy valued function on $[0, \infty[\times[0, \infty[$ into E. Suppose that the mappings $\tau \mapsto$ $F(\xi, \tau)=e^{-s \tau} u(\xi, \tau)$ and $\tau \mapsto G(\xi, \tau)=e^{-s \tau} u_{\tau}(\xi, \tau)$ are fuzzy Riemann integrable on $\left[0, \infty\left[\right.\right.$, for all $s \geq s_{0}$ for some $s_{0}>0$. Consider the following:

(a) If $u(\xi, \tau)$ is (i)-differentiable with respect to $\tau$, then

$$
\mathbf{L}_{\tau}\left[u_{\tau}(\xi, \tau)\right]=s \mathbf{L}_{\tau}[u(\xi, \tau)] \ominus u(\xi, 0),
$$

(b) If $u(x, \tau)$ is (ii)-differentiable with respect to $\tau$, then

$$
\mathbf{L}_{\tau}\left[u_{\tau}(\xi, \tau)\right]=(-u(\xi, 0)) \ominus(-s) \mathbf{L}_{\tau}[u(\xi, \tau)] .
$$

Proof. This is a direct result of Theorem 12, by fixing $\xi \geq 0$ and taking the Laplace transforms with respect to $\tau$.

\section{Fuzzy Laplace Transform Algorithm for First-Order Fuzzy Partial Differential Equations}

Our aim now is to solve the following first-order FPDE using the fuzzy Laplace transform method under strongly generalized differentiability:

$$
\begin{aligned}
u_{\xi}(\xi, \tau)+a u_{\tau}(\xi, \tau) & =f(\xi, \tau, u(\xi, \tau)), \\
u(\xi, 0) & =g(\xi), \\
u(0, \tau) & =h(\tau),
\end{aligned}
$$

where $u(\xi, \tau)$ is a fuzzy function of $\xi \geq 0, \tau \geq 0, a$ is a real constant, and $f(\xi, \tau, u), g(\xi)$, and $h(\tau)$ are fuzzy valued functions, such that $f(\xi, \tau, u)$ is linear with respect to $u$. For short, assume that $a \geq 0$ (case $a<0$ is similar).

By using fuzzy Laplace transform with respect to $\tau$, we get

$$
\mathbf{L}_{\tau}\left[u_{\xi}(\xi, \tau)\right]+a \mathbf{L}_{\tau}\left[u_{\tau}(\xi, \tau)\right]=\mathbf{L}_{\tau}[f(\xi, \tau, u(\xi, \tau))] .
$$

Therefore, we have to distinguish the following cases for solving (42): 
(a) Case 1: If $u$ is (i)-differentiable with respect to $\xi$ and $\tau$, then by Laplace transform

$$
\begin{aligned}
\mathscr{L} & {\left[\underline{u}_{\xi}(\xi, \tau, \alpha)\right]+a \mathscr{L}\left[\underline{u}_{\tau}(\xi, \tau, \alpha)\right] } \\
& =\mathscr{L}[\underline{f}(\xi, \tau, u(\xi, \tau))], \\
\mathscr{L} & {\left[\bar{u}_{\xi}(\xi, \tau, \alpha)\right]+a \mathscr{L}\left[\bar{u}_{\tau}(\xi, \tau, \alpha)\right] } \\
& =\mathscr{L}[\bar{f}(\xi, \tau, u(\xi, \tau))],
\end{aligned}
$$

where $f(\xi, \tau, u(\xi, \tau), \alpha)=\min \{f(\xi, \tau, v) / v \in(\underline{u}(\xi$, $\tau, \alpha), \bar{u}(\xi, \tau, \alpha))\}$ and $\bar{f}(\xi, \tau, u(\xi, \tau), \alpha)=\max \{f(\xi, \tau$, $v) / v \in(\underline{u}(\xi, \tau, \alpha), \bar{u}(\xi, \tau, \alpha))\}$.

Using Theorems 12 and 13 we get the following differential system:

$$
\begin{aligned}
\frac{\partial}{\partial \xi} & (\mathscr{L}[\underline{u}(\xi, \tau, \alpha)])+a s \mathscr{L}[\underline{u}(\xi, \tau, \alpha)] \\
& =a \underline{g}(\xi)+\mathscr{L}[\underline{f}(\xi, \tau, u(\xi, \tau))], \\
\frac{\partial}{\partial \xi} & (\mathscr{L}[\bar{u}(\xi, \tau, \alpha)])+a s \mathscr{L}[\bar{u}(\xi, \tau, \alpha)] \\
& =a \bar{g}(\xi)+\mathscr{L}[\bar{f}(\xi, \tau, u(\xi, \tau))]
\end{aligned}
$$

satisfying the following initial conditions:

$$
\begin{aligned}
& \mathscr{L}[\underline{u}(0, \tau, \alpha)]=\mathscr{L}[\underline{h}(\tau, \alpha)], \\
& \mathscr{L}[\bar{u}(0, \tau, \alpha)]=\mathscr{L}[\bar{h}(\tau, \alpha)] .
\end{aligned}
$$

Assume that this leads to

$$
\begin{aligned}
& \mathscr{L}[\underline{u}(\xi, \tau, \alpha)]=H_{1}(s, \alpha), \\
& \mathscr{L}[\bar{u}(\xi, \tau, \alpha)]=K_{1}(s, \alpha),
\end{aligned}
$$

where $\left(H_{1}(s, \alpha), K_{1}(s, \alpha)\right)$ is solution of system (44) under (45).

By the inverse Laplace transform we get

$$
\begin{aligned}
& \underline{u}(\xi, \tau, \alpha)=\mathscr{L}^{-1}\left[H_{1}(s, \alpha)\right], \\
& \bar{u}(\xi, \tau, \alpha)=\mathscr{L}^{-1}\left[K_{1}(s, \alpha)\right] .
\end{aligned}
$$

(b) Case 2: If $u$ is (i)-differentiable with respect to $\xi$ and (ii)-differentiable with respect to $\tau$, then by Theorems 12 and 13 we get the following differential system, satisfying the initial conditions (45):

$$
\begin{gathered}
\frac{\partial}{\partial \xi}(\mathscr{L}[\underline{u}(\xi, \tau, \alpha)])+a s \mathscr{L}[\bar{u}(\xi, \tau, \alpha)] \\
=a \bar{g}(\xi)+\mathscr{L}[\underline{f}(\xi, \tau, u(\xi, \tau))], \\
\frac{\partial}{\partial \xi}(\mathscr{L}[\bar{u}(\xi, \tau, \alpha)])+a s \mathscr{L}[\underline{u}(\xi, \tau, \alpha)] \\
=a \underline{g}(\xi)+\mathscr{L}[\bar{f}(\xi, \tau, u(\xi, \tau))] .
\end{gathered}
$$

Assume that this implies

$$
\begin{aligned}
& \mathscr{L}[\underline{u}(\xi, \tau, \alpha)]=H_{2}(s, \alpha), \\
& \mathscr{L}[\bar{u}(\xi, \tau, \alpha)]=K_{2}(s, \alpha),
\end{aligned}
$$

where $\left(H_{2}(s, \alpha), K_{2}(s, \alpha)\right)$ is solution of system (48) under (45).

Thus

$$
\begin{aligned}
& \underline{u}(\xi, \tau, \alpha)=\mathscr{L}^{-1}\left[H_{2}(s, \alpha)\right], \\
& \bar{u}(\xi, \tau, \alpha)=\mathscr{L}^{-1}\left[K_{2}(s, \alpha)\right] .
\end{aligned}
$$

(c) Case 3: If $u$ is (ii)-differentiable with respect to $\xi$ and (i)-differentiable with respect to $\tau$, then we get the following differential system, satisfying the initial conditions (45):

$$
\begin{aligned}
& \frac{\partial}{\partial \xi}(\mathscr{L}[\bar{u}(\xi, \tau, \alpha)])+\operatorname{as} \mathscr{L}[\underline{u}(\xi, \tau, \alpha)] \\
& =a \underline{g}(\xi)+\mathscr{L}[\underline{f}(\xi, \tau, u(\xi, \tau))], \\
& \frac{\partial}{\partial \xi}(\mathscr{L}[\underline{u}(\xi, \tau, \alpha)])+a s \mathscr{L}[\bar{u}(\xi, \tau, \alpha)] \\
& =a \bar{g}(\xi)+\mathscr{L}[\bar{f}(\xi, \tau, u(\xi, \tau))] .
\end{aligned}
$$

Assume that this implies

$$
\begin{aligned}
& \mathscr{L}[\underline{u}(\xi, \tau, \alpha)]=H_{3}(s, \alpha), \\
& \mathscr{L}[\bar{u}(\xi, \tau, \alpha)]=K_{3}(s, \alpha),
\end{aligned}
$$

where $\left(H_{3}(s, \alpha), K_{3}(s, \alpha)\right)$ is solution of system (51) under (45).

Therefore

$$
\begin{aligned}
& \underline{u}(\xi, \tau, \alpha)=\mathscr{L}^{-1}\left[H_{3}(s, \alpha)\right], \\
& \bar{u}(\xi, \tau, \alpha)=\mathscr{L}^{-1}\left[K_{3}(s, \alpha)\right] .
\end{aligned}
$$

(d) Case 4: If $u$ is (ii)-differentiable with respect to $\xi$ and $\tau$, then we get the following differential system, satisfying the initial conditions (45):

$$
\begin{aligned}
& \frac{\partial}{\partial \xi}(\mathscr{L}[\bar{u}(\xi, \tau, \alpha)])+a s \mathscr{L}[\bar{u}(\xi, \tau, \alpha)] \\
& =a \bar{g}(\xi)+\mathscr{L}[\underline{f}(\xi, \tau, u(\xi, \tau))], \\
& \frac{\partial}{\partial \xi}(\mathscr{L}[\underline{u}(\xi, \tau, \alpha)])+a s \mathscr{L}[\underline{u}(\xi, \tau, \alpha)] \\
& \quad=a \underline{g}(\xi)+\mathscr{L}[\bar{f}(\xi, \tau, u(\xi, \tau))] .
\end{aligned}
$$

Assume that this leads to

$$
\begin{aligned}
& \mathscr{L}[\underline{u}(\xi, \tau, \alpha)]=H_{4}(s, \alpha), \\
& \mathscr{L}[\bar{u}(\xi, \tau, \alpha)]=K_{4}(s, \alpha),
\end{aligned}
$$


where $\left(H_{4}(p, \alpha), K_{4}(p, \alpha)\right)$ is solution of system (54) under (45).

Hence

$$
\begin{aligned}
& \underline{u}(\xi, \tau, \alpha)=\mathscr{L}^{-1}\left[H_{4}(s, \alpha)\right], \\
& \bar{u}(\xi, \tau, \alpha)=\mathscr{L}^{-1}\left[K_{4}(s, \alpha)\right] .
\end{aligned}
$$

\section{Numerical Examples}

Example 1. Consider

$$
\begin{aligned}
u_{\xi}(\xi, \tau) & =3 u_{\tau}(\xi, \tau)+\xi, \\
u(\xi, 0, \alpha) & =3 \xi \cdot(\alpha, 2-\alpha)+\frac{\xi^{2}}{2}, \\
u(0, \tau, \alpha) & =\tau \cdot(\alpha, 2-\alpha),
\end{aligned}
$$

$$
\xi \geq 0, \tau \geq 0 \text {. }
$$

Case 1. If $u$ is (i)-differentiable with respect to $\xi$ and $\tau$, then by Laplace transform we get

$$
\begin{aligned}
\frac{\partial}{\partial \xi}(\mathscr{L}[\underline{u}(\xi, \tau, \alpha)])= & 3 s \mathscr{L}[\bar{u}(\xi, \tau, \alpha)]-9 \alpha \xi-\frac{3 \xi^{2}}{2} \\
& +\frac{\xi}{s}, \\
\frac{\partial}{\partial \xi}(\mathscr{L}[\bar{u}(\xi, \tau, \alpha)])= & 3 s \mathscr{L}[\underline{u}(\xi, \tau, \alpha)]+9(\alpha-2) \xi \\
& -\frac{3 \xi^{2}}{2}+\frac{\xi}{s} .
\end{aligned}
$$

This differential system satisfies the following initial conditions:

$$
\begin{aligned}
& \mathscr{L}[\underline{u}(0, \tau, \alpha)]=\mathscr{L}[\alpha \tau]=\frac{\alpha}{s^{2}}, \\
& \mathscr{L}[\bar{u}(0, \tau, \alpha)]=\mathscr{L}[(2-\alpha) \tau]=\frac{(2-\alpha)}{s^{2}} .
\end{aligned}
$$

Solving (58) under (59), we get

$$
\begin{aligned}
& \mathscr{L}[\underline{u}(\xi, \tau, \alpha)]=\frac{\left(3 \alpha \xi+\xi^{2} / 2\right)}{s}+\frac{\alpha}{s^{2}}, \\
& \mathscr{L}[\bar{u}(\xi, \tau, \alpha)]=\frac{\left((6-3 \alpha) \xi+\xi^{2} / 2\right)}{s}+\frac{2-\alpha}{s^{2}} .
\end{aligned}
$$

By the inverse Laplace transform we deduce

$$
\begin{aligned}
& \underline{u}(\xi, \tau, \alpha)=\alpha(3 \xi+\tau)+\frac{\xi^{2}}{2}, \\
& \bar{u}(\xi, \tau, \alpha)=(2-\alpha)(3 \xi+\tau)+\frac{\xi^{2}}{2} .
\end{aligned}
$$

The lengths of $u, u_{\xi}$, and $u_{\tau}$ are, respectively, given by

$$
\begin{aligned}
\text { len }(u(\xi, \tau, \alpha)) & =\bar{u}(\xi, \tau, \alpha)-\underline{u}(\xi, \tau, \alpha) \\
& =2(1-\alpha)(3 \xi+\tau) \geq 0, \\
\operatorname{len}\left(u_{\xi}(\xi, \tau, \alpha)\right) & =\bar{u}_{\xi}(\xi, \tau, \alpha)-\underline{u}_{\tau}(\xi, \tau, \alpha) \\
& =6(1-\alpha) \geq 0, \\
\operatorname{len}\left(u_{\tau}(\xi, \tau, \alpha)\right) & =\bar{u}_{\tau}(\xi, \tau, \alpha)-\underline{u}_{\tau}(\xi, \tau, \alpha) \\
& =2(1-\alpha) \geq 0 .
\end{aligned}
$$

So, this solution is valid for all $\xi \geq 0$ and $\tau \geq 0$.

Case 2. If $u$ is (i)-differentiable with respect to $\xi$ and (ii)differentiable with respect to $\tau$, then analogously

$$
\begin{aligned}
\underline{u}(\xi, \tau, \alpha)= & 2(\alpha-1)(\tau-3 \xi) H(\tau-3 \xi)+3 \alpha \xi \\
& +(2-\alpha) \tau+\frac{\xi^{2}}{2}, \\
\bar{u}(\xi, \tau, \alpha)= & 2(1-\alpha)(\tau-3 \xi) H(\tau-3 \xi)+3(2-\alpha) \xi \\
& +\alpha \tau+\frac{\xi^{2}}{2},
\end{aligned}
$$

where $H$ is the unit step function or the Heaviside function:

$$
H(\zeta)= \begin{cases}1 & \zeta \geq 0 \\ 0 & \zeta<0\end{cases}
$$

Therefore, this solution $u$ is valid only over $\Delta=\{(\xi, \tau) \mid$ $\xi \geq 0, \tau \geq 0, \tau \leq 3 \xi\}$.

Case 3. If $u$ is (ii)-differentiable with respect to $\xi$ and (i)differentiable with respect to $\tau$, then similarly

$$
\begin{aligned}
\underline{u}(\xi, \tau, \alpha)= & 2(\alpha-1)(\tau-3 \xi) H(\tau-3 \xi)+3 \alpha \xi \\
& +(2-\alpha) \tau+\frac{\xi^{2}}{2}, \\
\bar{u}(\xi, \tau, \alpha)= & 2(1-\alpha)(\tau-3 \xi) H(\tau-3 \xi)+3(2-\alpha) \xi \\
& +\alpha \tau+\frac{\xi^{2}}{2} .
\end{aligned}
$$

As in Case 2, one can verify that this solution is valid only over $\Delta^{\prime}=\{(\xi, \tau) \mid \xi \geq 0, \tau \geq 0, \tau \geq 3 \xi\}$.

Case 4. If $u$ is (ii)-differentiable with respect to $\xi$ and $\tau$, then

$$
\begin{aligned}
& \underline{u}(\xi, \tau, \alpha)=\alpha(3 \xi+\tau)+\frac{\xi^{2}}{2}, \\
& \bar{u}(\xi, \tau, \alpha)=(2-\alpha)(3 \xi+\tau)+\frac{\xi^{2}}{2} .
\end{aligned}
$$

One can verify that function $u$ is not (ii)-differentiable with respect to either $\xi$ or $\tau$.

So, no solution exists in this case. 
Example 2. Consider

$$
\begin{aligned}
u_{\xi}(\xi, \tau) & =u_{\tau}(\xi, \tau), \\
u(\xi, 0, \alpha) & =\cos (\xi) \cdot(\alpha, 2-\alpha), \\
u(0, \tau, \alpha) & =\cos (\tau) \cdot(\alpha, 2-\alpha),
\end{aligned}
$$

$$
\xi \geq 0, \tau \geq 0 .
$$

Case 1. If $u$ is (i)-differentiable with respect to $\xi$ and $\tau$, then, by application of the algorithm above, one obtains

$$
\begin{aligned}
& \underline{u}(\xi, \tau, \alpha)=\alpha \cos (\xi+\tau), \\
& \bar{u}(\xi, \tau, \alpha)=(2-\alpha) \cos (\xi+\tau) .
\end{aligned}
$$

The lengths of $u, u_{\xi}$, and $u_{\tau}$ are, respectively, given by

$$
\begin{aligned}
\text { len }(u(\xi, \tau, \alpha)) & =2(1-\alpha) \cos (\xi+\tau), \\
\operatorname{len}\left(u_{\xi}(\xi, \tau, \alpha)\right) & =-2(1-\alpha) \sin (\xi+\tau), \\
\operatorname{len}\left(u_{\xi}(\xi, \tau, \alpha)\right) & =-2(1-\alpha) \sin (\xi+\tau) .
\end{aligned}
$$

So, this solution is valid for all $\xi \geq 0, \tau \geq 0$ : $\xi+\tau \in$ $[3 \pi / 2+2 k \pi, 2 \pi+2 k \pi], k \in \mathbb{Z}$.

Case 2. If $u$ is (i)-differentiable with respect to $\xi$ and (ii)differentiable with respect to $\tau$, therefore

$$
\begin{aligned}
& \underline{u}(\xi, \tau, \alpha)=(\alpha-1) \cos (\xi-\tau)+\cos (\xi+\tau), \\
& \bar{u}(\xi, \tau, \alpha)=(1-\alpha) \cos (\xi-\tau)+\cos (\xi+\tau) .
\end{aligned}
$$

Then, this solution is valid for all $\xi \geq 0, \tau \geq 0: \xi-\tau \in$ $[3 \pi / 2+2 k \pi, 2 \pi+2 k \pi], k \in \mathbb{Z}$.

Case 3. If $u$ is (ii)-differentiable with respect to $\xi$ and (i)differentiable with respect to $\tau$, then analogously

$$
\begin{aligned}
& \underline{u}(\xi, \tau, \alpha)=(\alpha-1) \cos (\xi-\tau)+\cos (\xi+\tau), \\
& \bar{u}(\xi, \tau, \alpha)=(1-\alpha) \cos (\xi-\tau)+\cos (\xi+\tau) .
\end{aligned}
$$

Hence, this solution is valid for all $\xi \geq 0, \tau \geq 0$ : $\xi-\tau \in$ $[2 k \pi, \pi / 2+2 k \pi], k \in \mathbb{Z}$.

Case 4. If $u$ is (ii)-differentiable with respect to $\xi$ and $\tau$, then similarly

$$
\begin{aligned}
& \underline{u}(\xi, \tau, \alpha)=\alpha \cos (\xi+\tau), \\
& \bar{u}(\xi, \tau, \alpha)=(2-\alpha) \cos (\xi+\tau) .
\end{aligned}
$$

So, this solution is valid for all $\xi \geq 0, \tau \geq 0$ : $\xi+\tau \in$ $[2 k \pi, \pi / 2+2 k \pi], k \in \mathbb{Z}$.

\section{Conclusion}

Theorems of continuity and differentiability for a fuzzy function defined via a fuzzy improper Riemann integral are proved which are used to prove some results concerning fuzzy Laplace transform. Then, using Laplace transform method, the solutions for some linear fuzzy partial differential equations (FPDEs) of first order are given. For future research, one can apply this method to solve FPDEs of high order.

\section{Conflict of Interests}

The authors declare that there is no conflict of interests regarding the publication of this paper.

\section{References}

[1] H.-C. Wu, "The improper fuzzy Riemann integral and its numerical integration," Information Sciences, vol. 111, no. 1-4, pp. 109-137, 1998.

[2] T. Allahviranloo and M. B. Ahmadi, "Fuzzy Laplace transforms," Soft Computing, vol. 14, no. 3, pp. 235-243, 2010.

[3] S. Salahshour, M. Khezerloo, S. Hajighasemi, and M. Khorasany, "Solving fuzzy integral equations of the second kind by fuzzy laplace transform method," International Journal of Industrial Mathematics, vol. 4, no. 1, pp. 21-29, 2012.

[4] S. Salahshour and T. Allahviranloo, "Applications of fuzzy Laplace transforms," Soft Computing, vol. 17, no. 1, pp. 145-158, 2013.

[5] E. ElJaoui, S. Melliani, and L. S. Chadli, "Solving second-order fuzzy differential equations by the fuzzy Laplace transform method," Advances in Difference Equations, vol. 2015, article 66, 14 pages, 2015.

[6] O. Kaleva, "Fuzzy differential equations," Fuzzy Sets and Systems, vol. 24, no. 3, pp. 301-317, 1987.

[7] M. L. Puri and D. A. Ralescu, "Fuzzy random variables," Journal of Mathematical Analysis and Applications, vol. 114, no. 2, pp. 409-422, 1986.

[8] I. J. Rudas, B. Bede, and A. L. Bencsik, "First order linear fuzzy differential equations under generalized differentiability," Information Sciences, vol. 177, no. 7, pp. 1648-1662, 2007.

[9] B. Bede and S. G. Gal, "Generalizations of the differentiability of fuzzy-number-valued functions with applications to fuzzy differential equations," Fuzzy Sets and Systems, vol. 151, no. 3, pp. 581-599, 2005.

[10] Y. Chalco-Cano and H. Román-Flores, "On new solutions of fuzzy differential equations," Chaos, Solitons \& Fractals, vol. 38, no. 1, pp. 112-119, 2008. 


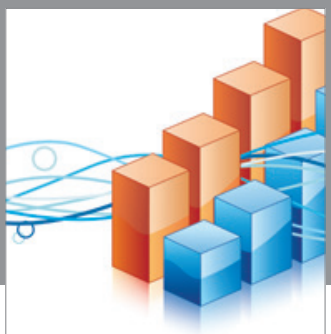

Advances in

Operations Research

vatem alat4

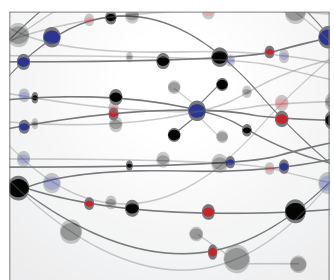

\section{The Scientific} World Journal
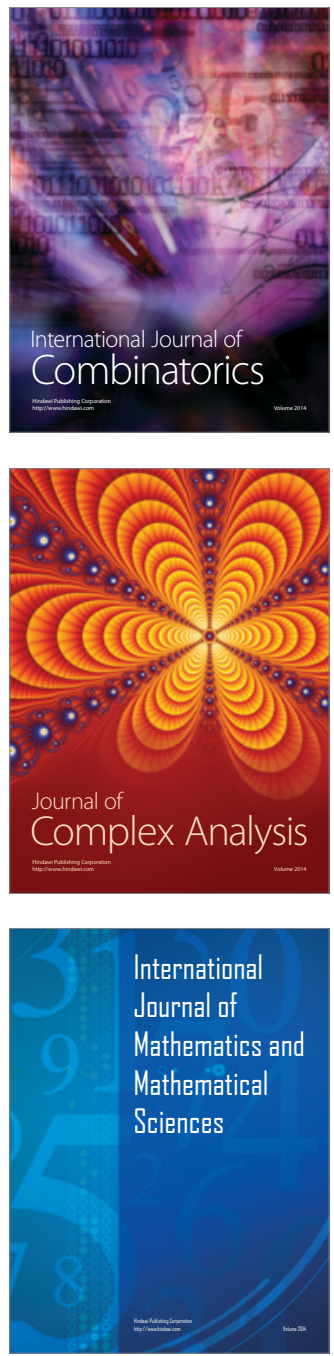
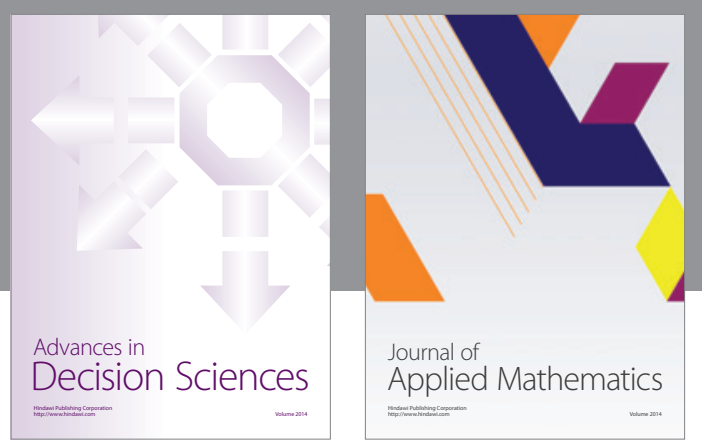

Algebra

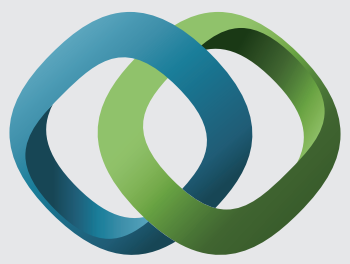

\section{Hindawi}

Submit your manuscripts at

http://www.hindawi.com
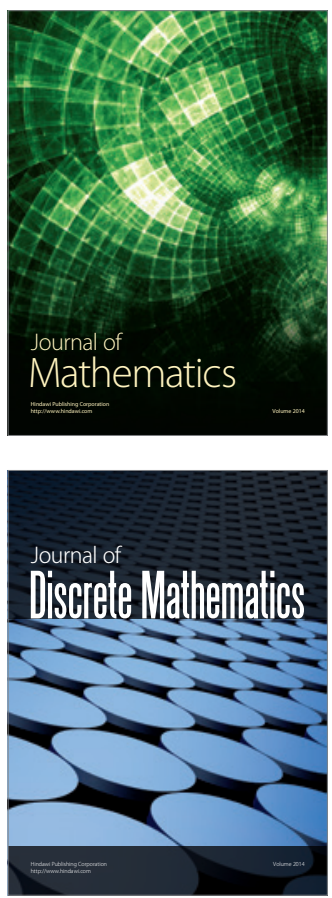

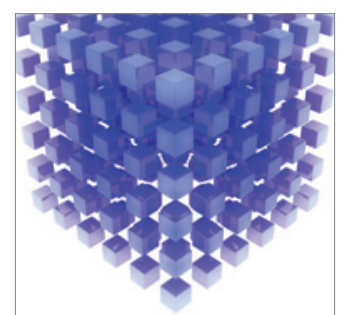

Mathematical Problems in Engineering
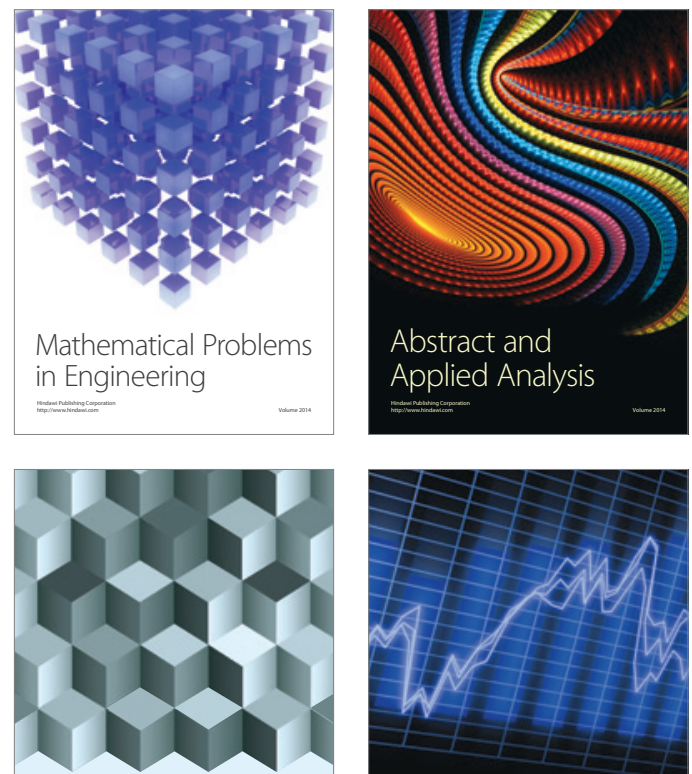

Journal of

Function Spaces

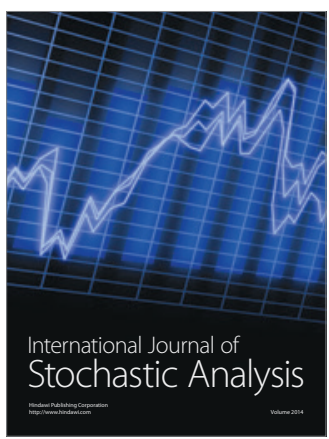

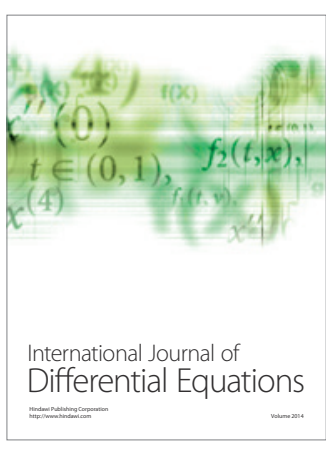
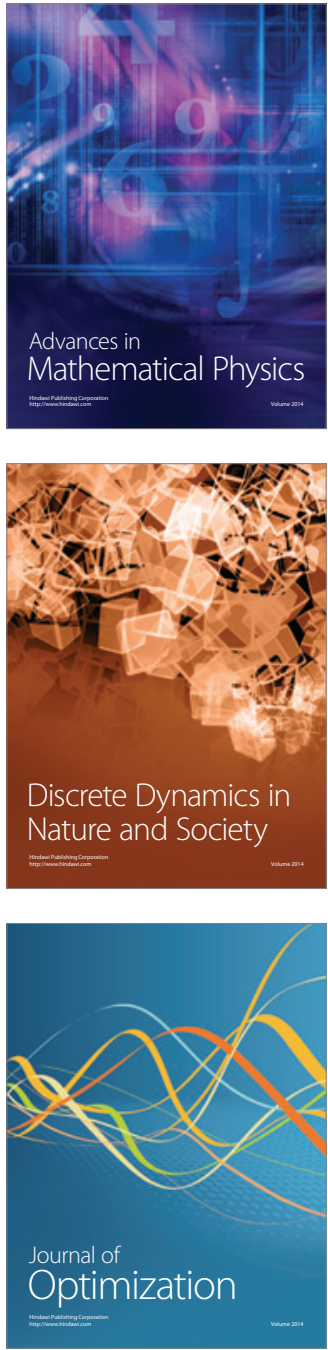